\title{
Post-COVID-19 exacerbation of fibrodysplasia ossificans progressiva with multiple flare-ups and extensive heterotopic ossification in a 45-year-old female patient
}

\author{
Lovorka Grgurevic $^{1,2}$ (D) $\cdot$ Rudjer Novak $^{2}$ (D) $\cdot$ Stela Hrkac $^{2}$ (D) $\cdot$ Grgur Salai $^{2}$ (D) $\cdot$ Simeon Grazio $^{3}$ (D)
}

Received: 8 May 2021 / Accepted: 3 June 2021 / Published online: 10 June 2021

(c) The Author(s) 2021

\begin{abstract}
Fibrodyplasia ossificans progressiva (FOP) is a rare hereditary disease, which has a variable course characterized by occasional flare-ups of heterotopic ossification (HO) in soft tissues that are followed by swelling, stiffness, pain and warmth. Here, we report for the first time a case of a 45-year-old female patient with known FOP recovering from COVID-19 with disease progression potentially linked with the viral illness. In December 2020 the patient contracted a mild form of COVID-19 infection without need for hospital admission. Since January 2021, the patient felt unwell, with occasional abdominal pain which progressively intensified. In March 2021 she presented with new onset of HO, complaining of pain, swelling and thickening sensation in the lower abdomen and left part of the neck. Computerized tomography (CT) and cytokine analysis were performed. CT scan revealed new heterotopic bone formation in multiple soft tissue areas of the neck indicating clear radiological progression. Radiotherapy, which has proven to be an efficient tool to control $\mathrm{HO}$ in this patient, was not able to halt HO formation after COVID-19 infection. Cytokine analysis of a plasma sample obtained during a flare-up after COVID19 infection showed a significantly elevated pro-inflammatory cytokines compared to a flare-up panel prior to infection. Of the 23 analyzed levels of cytokines, a staggering number of 21 were above normal levels. This case is the first confirmation of uncontrolled post-COVID-19 effects in a FOP patient, which manifested with flare-ups followed by progressive HO, possibly caused by a thus far, never described form of post-COVID syndrome.
\end{abstract}

Keywords Myositis ossificans · COVID-19 · Cytokines · Heterotopic ossification

\section{Introduction}

Fibrodysplasia ossificans progressiva (FOP) is a rare, but debilitating disease that affects 1:2,000,000 people worldwide. The disease causes bone formation in muscles and connective tissues, effectively entombing the patient in a

Stela Hrkac and Grgur Salai contributed equally.

Lovorka Grgurevic

lovorka.grgurevic@mef.hr

1 Department of Anatomy, School of Medicine, University of Zagreb, Zagreb, Croatia

2 Center for Translational and Clinical Research, Department of Proteomics, School of Medicine, University of Zagreb, Salata 11, Zagreb, Croatia

3 Department of Rheumatology, Physical and Rehabilitation, Medicine University Hospital Center "Sestre Milosrdnice", Zagreb, Croatia "second skeleton". The earliest skeletal malformations that are indicators of FOP can be present at birth, such as inward curving of the great toe. The disease progresses variably, and is characterized by episodes of exacerbation (flareups) with heterotopic ossification (HO) in soft tissues. HO appears spontaneously or after trauma to skeletal muscles and connective tissues and is characterized by swelling, stiffness, pain and warmth. Some lesions regress spontaneously, but most of them mature by an endochondral ossification pathway [1]. Although most cases are sporadic, the disease can be inherited through an autosomal dominant mutated ACVR1 gene, also known as Alk-2. This gene encodes an activin A receptor type I for bone morphogenetic proteins (BMP), which are members of the TGF $\beta$ superfamily [2]. Although animal studies have shown that Activin A inhibition could be highly effective in blocking progressive $\mathrm{HO}$, this mutation alone is not sufficient for $\mathrm{HO}$, since disease flare-ups are triggered by inflammation and activation of the innate immune system [3]. Studies have shown that 
innate immunity and cytokines are of great importance for $\mathrm{HO}$ occurrence and progression, furthermore, various cytokines have been implicated in $\mathrm{HO}$ pathogenesis $[4,5]$. Previous research hypothesized that for the induction of $\mathrm{HO}$ in FOP patients an increase in at least one pro-inflammatory cytokine is both essential and sufficient to trigger the entire process of inflammatory cell influx [6]. Coronavirus disease (COVID-19) is caused by SARS-CoV-2 and represents a global public health concern [7]. It can present with various symptoms and degrees of severity, some of which can be life-endangering and even fatal [8].

In this report, we describe the case of a 45 -year-old female patient with FOP who suffered from a mild form of COVID-19 infection after which she experienced intense FOP exacerbation (i.e., flare-up). This case report is complemented by patient's plasma cytokine profile analysis and literature review on the association of FOP and HO with COVID-19. To the best of our knowledge, this is the first report of a FOP patient recovering from COVID-19 and the first time COVID-19 convalescence has been linked to FOP progression.

\section{Case presentation}

A 45-year-old female patient with FOP presented to the Rheumatology Outpatient Clinic in March 2021, complaining of pain and swelling of the lower abdomen and left part of the neck. The patient was diagnosed with FOP at the age of four when a red, painful gristle appeared on her back, which later turned into bone (Fig. 1). This was followed by progressive ossification of the paravertebral muscles, leading to kyphoscoliosis, making her unable to erect herself by the age of ten. During her teenage years, occasional use of etidronate and methylprednisolone seemed to somewhat slow down formation of new $\mathrm{HO}$ foci. However, at the age of 25 (year 2000), new HO occurred in her right arm and both knees, followed by development of eating difficulties due to the affection of masticatory muscles (Fig. 1). The patient herself reported subjective improvement of $\mathrm{HO}$ shortly after diagnostic X-ray, which led to the decision to start radiotherapy treatment. At the age of 27 , she received a dose of $10 \mathrm{~Gy}$ (delivered in five fractions) on her right arm and 8 Gy (delivered in two fractions) on both knees. This halted HO progression over the following 2 years, as confirmed by X-ray. Keeping with the progressive nature of FOP, in 2004 new HO foci started developing. Radiation therapy was again attempted several times until 2010 on her right femur, infraclavicular region, neck and right hemithorax, where she was treated with $2 \mathrm{~Gy}$ in two, $6 \mathrm{~Gy}$ in six, $4 \mathrm{~Gy}$ in four, and $3 \mathrm{~Gy}$ in three fractions. The patient positively responded to the therapy and was well over the period of 9 years. However, another flare-up event occurred in 2019, with swelling and $\mathrm{HO}$ in the anterior part of the neck. A cytokine analysis was performed along with routine laboratory processing and radiotherapy treatment (4 Gy in 4 fractions), which resulted in immediate alleviation of bone formation and good function (Fig. 1).

In December 2020 the patient and her household family members contracted the COVID-19 infection, as confirmed by RT-PCR test on a sample obtained from a nasopharyngeal (NP) swab for SARS-CoV-2. Main symptoms were mild fever $\left(\max 37.5^{\circ} \mathrm{C}\right)$, mild cough, fatigue and sweating. She was treated as an outpatient with azithromycin and paracetamol and over the next 2 weeks, she recovered completely without the need for hospital admission and with negative control NP swab test for SARS-CoV-2. FOP exacerbation had restarted approximately 4 weeks post-COVID-19 convalescence and has thus continued steadily (Fig. 1). Since January 2021, the patient did not feel well, which was accompanied by changes in blood pressure and fatigue, with occasional abdominal pain attributed to a possible urinary tract infection. In February 2021, abdominal pain progressively intensified after she was examined by an abdominal

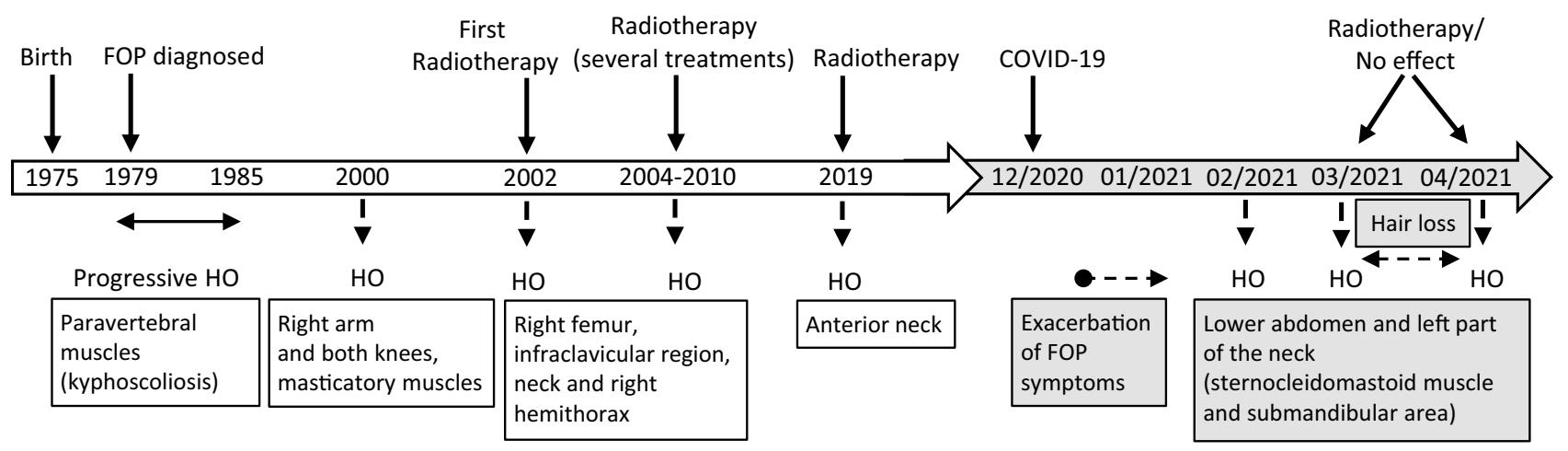

Fig. 1 Progression timeline in Patient 1 with FOP. Disease progression is depicted chronologically according to timepoints of HO occurrence and their respective location, as well as timepoints of radiother- apy. Events and disease exacerbation after COVID-19 infection are highlighted in grey 
surgeon on several occasions for suspected acute abdomen. A month later, she presented with new onset of HO, complaining of pain, swelling and thickening of tissues in the lower abdomen and left part of the neck (sternocleidomastoid muscle). Upon admission, a clear progression of $\mathrm{HO}$ was observed on CT scan of the same neck area from September 2020 (Fig. 2). Radiotherapy treatment was used again on the lower abdomen (4 Gy in four fractions) and on the left part of the patient's neck (4 Gy in two fractions). Unfortunately, the treatment was not successful as forewarned by the patient's residual feeling of uninterrupted swelling. In April 2021 , the patient reported a new area of progression in the left submandibular area, marginal to previous radiotherapy field. A CT scan confirmed new HO located in the oropharynx, indicating clear radiological progression (Fig. 2). She again received $4 \mathrm{~Gy}$ in 2 fractions to the affected area, however, with no apparent clinical response. Neck movement is currently completely restricted, as well as movement of other parts of the patient's body. Unfortunately, if such FOP progression persists, she is expected to develop inability to swallow and be respiratory compromised.

Radiotherapy was proven as an efficient tool to control $\mathrm{HO}$ in this patient. In addition to radiotherapy, during periods of flare-ups and $\mathrm{HO}$, the patient was treated with corticosteroids (methylprednisolone) up to doses of $64 \mathrm{mg}$ per day with rapid decrease in dosage. She also continuously takes several anti-inflammatory and analgesic medications: a selective COX-2 inhibitor, a combination of paracetamol and opioid analgesic, a leukotriene and a histamine receptor antagonist. However, after SARS-CoV-2 infection, FOP has progressed, and radiotherapy has been unable to halt
HO formation. Pathophysiological mechanisms to explain these observations are lacking. No damage or dysfunction of other vital organs was found in the patient after COVID-19 infection. Among the usual laboratory blood tests performed before and after COVID-19 infection, the only difference observed was in the values of C-reactive protein (CRP). Slightly elevated levels of CRP were observed in April 2021 (8.7 mg/L; reference values $<5 \mathrm{mg} / \mathrm{L}$ ) in comparison to September 2020 and March 2021, which were within the reference interval $(5.1 \mathrm{mg} / \mathrm{mL})$. Increased hair loss was observed 3 months after infection as part of post-COVID-19 syndrome; no other signs or symptoms were observed or reported by the patient.

Being aware of the rarity of her disease, this patient frequently contributes towards understanding of FOP by providing plasma samples for scientific purposes which are obtained for the purposes of evaluation and treatment during flare-ups (namely, she only consents her blood to be drawn when it is absolutely indicated for fear that the microtrauma of venipuncture might trigger HO). A panel of 23 pro- and anti-inflammatory mediators was used to screen her plasma before and after COVID-19 infection. The results were compared to three control samples: two female FOP patients in remission that were not COVID-19 positive and a plasma pool of ten healthy women of the same age group with no bone disorders. This study was approved by the institutional ethics committee and all participants signed an informed consent form. Blood samples were drawn into standard vacuette citrate tubes with special care as not to induce $\mathrm{HO}$ by needle insertion. Participants' plasma samples were screened for differences in cytokine levels using the human cytokine

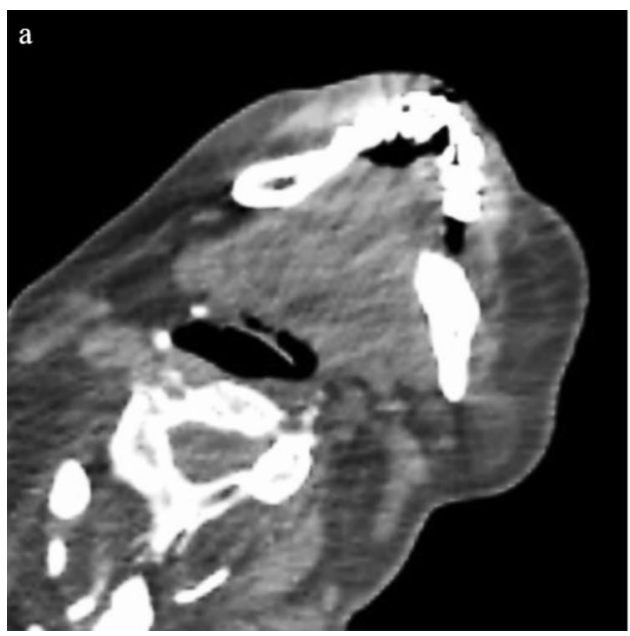

Fig. 2 Axial computed tomography (CT) images of the FOP patient taken pre- (a) and post-COVID-19 infection (b). New foci of heterotopic ossification surounding the oropharynx at the level of cervical vertebrae No 5 (C5) appeared after COVID-19 infection convalescence (white arrows). Due to disease progression resulting in severe

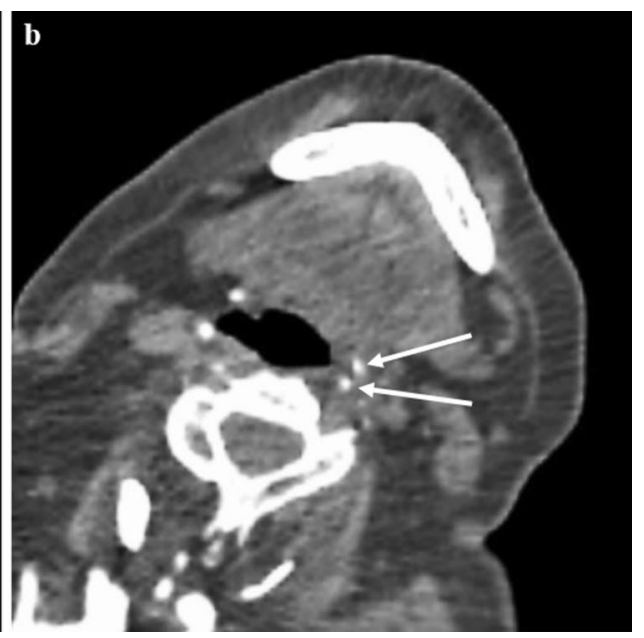

ambulatory restriction, movement restraint and fixed mandibula, there is a difference in neck position compared to baseline scan (CT machine: Toshiba Aquilion Large Bore 16 slices CT scanner, Toshiba Medical Systems Corporation, Tokyo, Japan) 
antibody array (\#ab133996, Abcam) and analyzed using the Chemi Doc imaging system (BioRad) according to manufacturer's recommendations. Quantification of chemiluminescent signals was performed with ImageJ 1.52a software (NIH) [9]. The cytokine expression levels are presented as fold change relative to the expression levels of the healthypool control sample (Fig. 3). Plasma cytokine expression during a flare-up in the period after COVID-19 infection, showed strikingly higher levels of most of the analyzed cytokines, as compared to the pre-COVID-19 panel of the same patient (Fig. 3c-e).

\section{Search strategy and literature overview}

We searched the PubMed and Scopus databases for original articles, reviews, letters, short communications and notes in English from 1971 for PubMed and from 2004 for Scopus to the latest date, which was May 2021 for both. Our search strategy included looking for titles, abstracts, and keywords, using the following terms: ("Fibrodysplasia ossificans progressiva" OR "FOP") AND ("COVID-19" OR "SARSCoV-2"), including a search of PubMed by the aforementioned MeSH terms [10]. The search was further expanded by replacing either of the terms: "COVID-19" with "viral illness" or "FOP" with "HO" (heterotopic ossification), in all combinations. The references in each selected study were reviewed to identify other relevant articles. The search results are shown in Table 1.

\section{Results}

Our search for FOP and COVID-19-related biomedical literature, conducted as previously described, did not yield any results. Upon furthering our search strategy, three articles were found (Table 1): two that relate HO to COVID-19 and another that connects FOP flare-ups with a viral illness (Influenza type B).

\section{Discussion}

FOP is recognized as one of the most debilitating diseases known to medicine. In addition to its severe and often unpredictable clinical course, there is, despite extensive research, no specific curative or preventive treatment. The exact mechanism and triggers for HO in FOP remain largely unclear. It is recognized that the ACVR1 mutation leads to enhanced Smad1/5/8 signaling and altered responsiveness to canonical (BMP) and non-canonical (Activin A) ligands [14]. However, ACVR1 mutation and Activin A are not solely responsible (or sufficient) for HO to occur. Previous research shows that involvement of immune cells, such as macrophages and mast cells, as well as various cytokines are of great importance in the process of $\mathrm{HO}$ [5]. In a previous study of plasma cytokine levels in FOP patients, it was hypothesized that RANTES (Regulated on activation, normal T-cell expressed and secreted/CCL5) might be a potential trigger and/or indicator of HO [6]. Cytokine analysis of a sample obtained during a flare-up prior to COVID-19 infection from the same patient, showed a significantly lower amount of elevated pro-inflammatory cytokines compared to the post-COVID-19 panel. These laboratory findings were also reflected in better pre-COVID-19 reaction to radiotherapy, which was followed by symptom alleviation. In our patient's post-COVID-19 cytokine panel, most notable elevations were found in MCP-1, RANTES and IL-13, which are cytokines with emerging roles in bone remodeling and cortical bone formation [15-17]. We also found a significant elevation of plasma IL-5, which has been reported to cause ectopic bone formation in animal models [18]. Of the 23 analyzed cytokines, a staggering number of 21 were above normal (healthy control) expression levels. Among them, IL-1, IL-6, IL-8, Interferon- $\gamma$, TNF $\alpha$ and GM-CSF were prominently increased. Limitations of the cytokine analysis we conducted include the lack of remission phase sample of our patient, inability to precisely quantify cytokine concentrations and possibility that crucial cytokines for understanding of the observed $\mathrm{HO}$ are not part of the cytokine panel kit we employed. In spite of these limitations, our findings suggest an upward trend from our patient's "baseline" flareup values. We suggest that such cytokine hyperproduction is the result of an imbalance in natural regulation mechanisms that are used to eliminate pathogens and avoid an exaggerated immune response. In this case, an overabundance of cytokines probably initiated marked worsening of the underlying disease. We observed no other systemic manifestations or damage to vital organs that are usually present in conditions with similar cytokine disturbances [19]. We hypothesize that COVID-19 might have acted as a longterm trigger for disease exacerbation, i.e., as an inductor of flare-ups which progressed to sites of HO. The effects of influenza-like illness on FOP patients have been documented before, as patients reported flare-ups which occurred after influenza-like symptoms [11]. Interestingly, in patients of the aforementioned study, the neck and trunk were commonly affected sites - which were also affected in our FOP patient after COVID-19 infection. However, these reported flare-ups occurred within days of viral illness onset, whereas our patient experienced disease worsening 4 weeks after COVID-19 infection convalescence with no improvement to this moment. This might be due to the specific nature of this disease, namely, its associated post-COVID-19 syndrome, which occurs beyond 4 weeks from the onset of symptoms. Tissue damage and innate immune response with inflammatory cytokine production may contribute to the various 
a

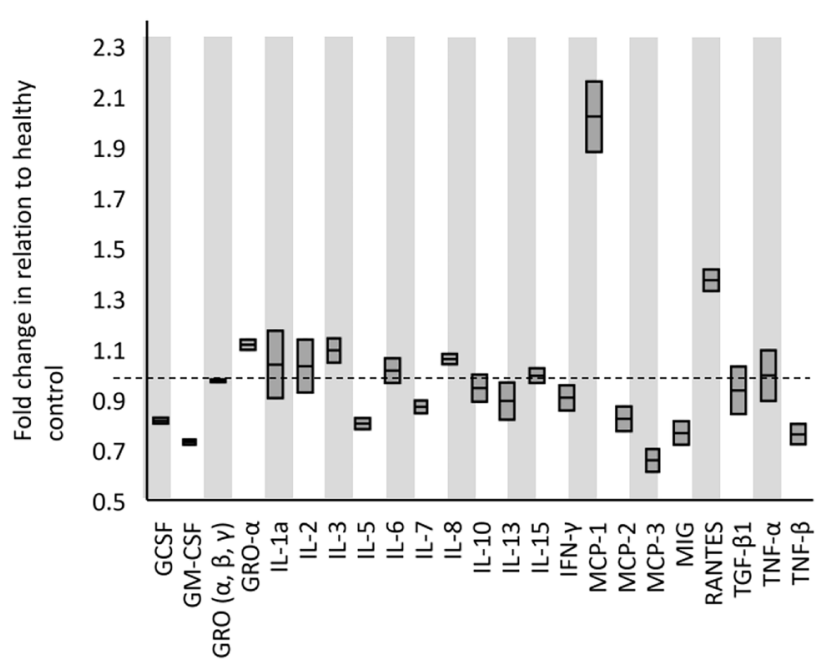

C

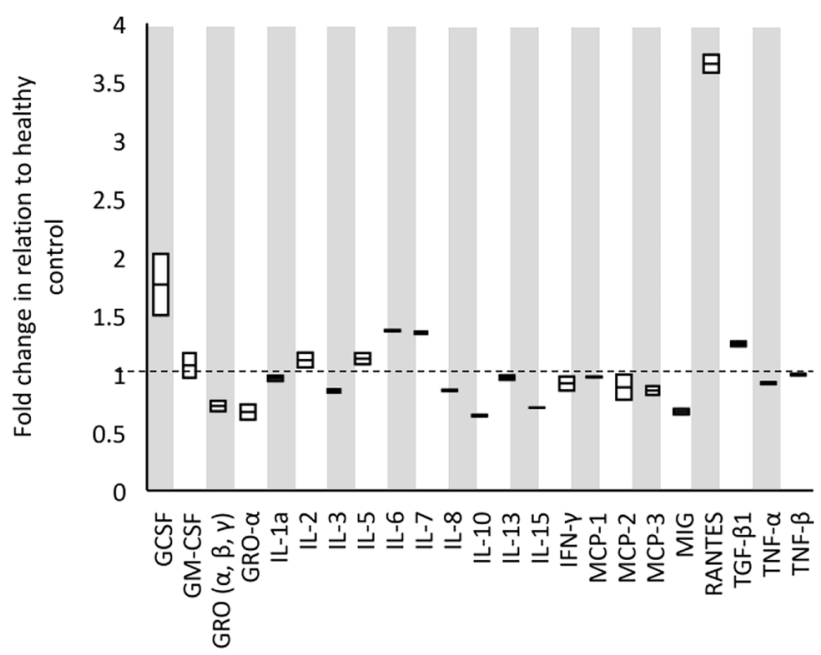

b

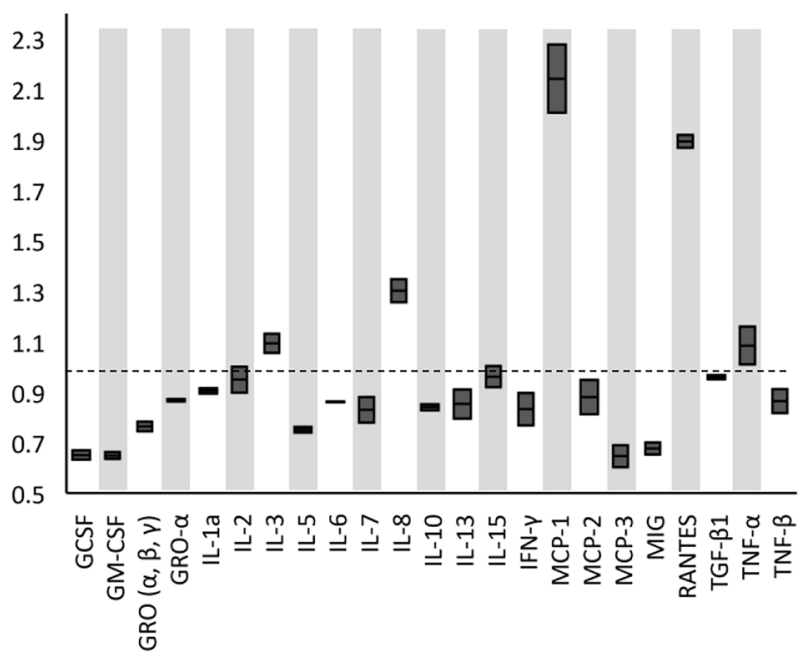

d

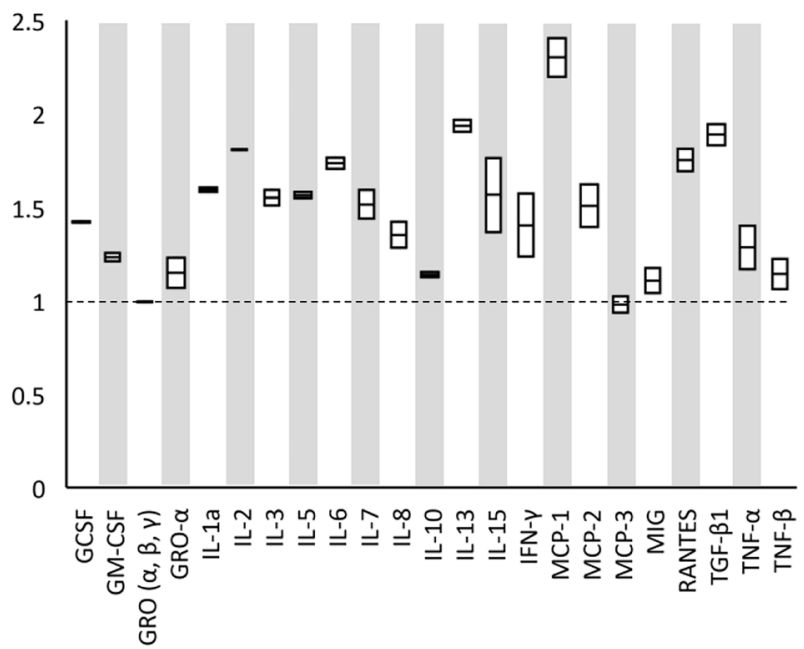

e

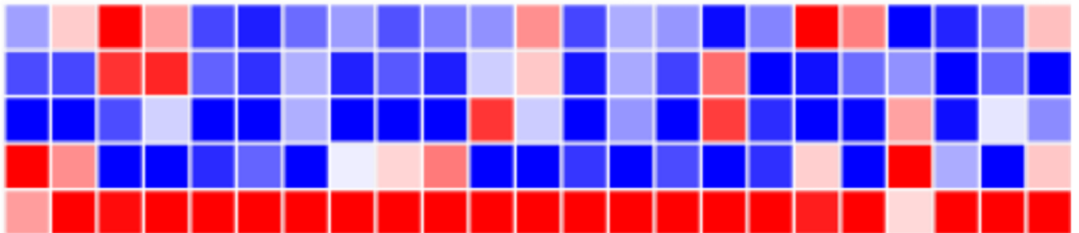

healthy control

FOP remission 1

FOP remission 2

FOP flare-up pre-COVID19

FOP flare-up post-COVID19

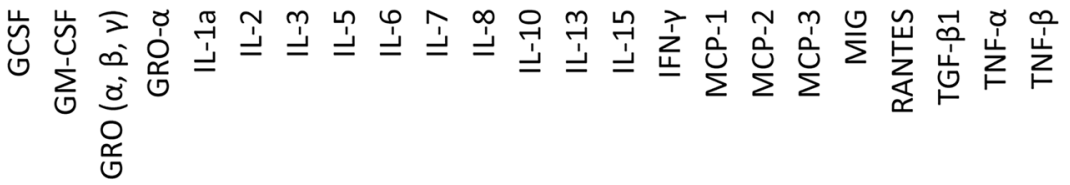

Fig. 3 Cytokine profiles of FOP patients presented as relative fold change in relation to healthy control: a FOP patient 2 (control) during a disease remission phase; b FOP patient 3 (control) during a disease remission phase; c FOP patient 1 (presented in the case) during a disease flare-up (sample obtained prior to COVID-19 infection); d

FOP patient 1 (presented in the case) during a disease flare-up (sample obtained 3 months after COVID-19 infection); e Heat map depicting cytokine expression profiles of healthy controls and FOP patients during remission (FOP remission 2 and 3, in comparison to FOP preand post-COVID-19 flare-ups in patient 1 
Table 1 Relevant articles pertaining to $\mathrm{FOP} / \mathrm{HO}$ and COVID-19/viral illness

\begin{tabular}{|c|c|c|c|c|c|c|c|}
\hline Reference & Article type & $N^{\mathrm{a}}$ & Sex & Age & Viral illness & $\begin{array}{l}\text { Underly- } \\
\text { ing FOP }\end{array}$ & $\mathrm{HO}^{\mathrm{e}}$ site \\
\hline Scarlett et al. [11] & Survey & 123 & $\begin{array}{l}53 \mathrm{Mb} \\
70 \mathrm{~F}^{\mathrm{c}}\end{array}$ & $3-72$ & Influenza-like & Yes & $\begin{array}{l}\text { Neck, back, } \\
\text { trunk, groin, } \\
\text { legs }\end{array}$ \\
\hline Meyer et al. [12] & Case-series & 4 & $4 \mathrm{M}$ & $39-74$ & COVID-19 & No & Shoulders \\
\hline Aziz et al. [13] & Case-series & 2 & $1 \mathrm{~F}$ & 43,51 & COVID-19 & No & Hip, shoulder \\
\hline
\end{tabular}

${ }^{a} N$ number of participants

${ }^{\mathrm{b}} M$ male

${ }^{\mathrm{c}} F$ female

${ }^{\mathrm{d}}$ FOP fibrodysplasia ossificans progressive

${ }^{\mathrm{e}} \mathrm{HO}$ heterotopic ossification sequelae of this syndrome [20]. We argue that tissue damage caused by post-COVID-19 syndrome might be the potential trigger of our patient's notable disease progression, as well as the associated elevation of inflammatory cytokines. Notably, several instances of non-FOP-related HO after severe forms of COVID-19 have been reported, which further supports our hypothesis $[12,13]$.

\section{Conclusion}

This case is the first case report of uncontrolled postCOVID-19 effects in a FOP patient, which manifested with consecutive disease flare-ups followed by progressive HO. The episode was possibly caused by a, thus far, never described form of post-COVID syndrome, which regularly affects the most sensitive and already disturbed parts of the body (locus minoris resistentiae). Prolonged ectopic ossification after COVID-19 infection, with marked changes in the cytokine profile of our patient, may point towards potential mechanisms contributing to the pathophysiology of post-acute COVID-19 syndrome, which in this case might be accompanied by aberrations of the innate and adaptive immune system. Understanding of these mechanisms is limited and further study is needed to improve FOP patient care during the COVID-19 pandemic and beyond.

Acknowledgements We thank all the participating FOP patients on their significant contribution to science, their active involvement and a great desire to help in finding appropriate therapeutic solution for this rare bone disease. We thank MD, PhD Jure Murgic for helping with additional clinical insights. This work was in part supported by the Scientific Center of Excellence for Reproductive and Regenerative Medicine (project "Reproductive and regenerative medicine-exploration of new platforms and potentials", Grant Agreement KK.01.1.1.01.0008) which is funded by the European Union through the European Regional Development Fund, so we thank them for the support shown to our program.

Author contribution LG-conceptualization, writing - original draft, review and editing; $\mathrm{RN}$ - formal analysis, writing — review and editing;
SH—writing — review and editing; GS—-data curation, writing — review and editing; SG-resources, writing — review and editing.

Funding University project "Determination of the differences in the protein profile and incidence of inflammatory mediators in the blood and urine of patients with FOP, and bone induction assay using the egg extract in cell culture" and Scientific Center of Excellence for Reproductive and Regenerative Medicine (project "Reproductive and regenerative medicine-exploration of new platforms and potentials", Grant Agreement KK.01.1.1.01.0008 which is funded by the European Union through the European Regional Development Fund.

Availability of data and materials The data sets used and/or analyzed during the current study are available from the corresponding author on reasonable request.

\section{Declarations}

Conflict of interest Lovorka Grgurevic, Rudjer Novak, Stela Hrkac, Grgur Salai and Simeon Grazio declare that they have no conflict of interest.

Consent to participate Informed consent for study participation was obtained from all individual participants included in the study.

Consent for publication Informed consent for journal publication was obtained from the participants described in the study, including permission for publication of images.

Ethics approval and consent to participate All procedures performed in studies involving human participants were in accordance with the ethical standards of the institutional and/or national research committee and with the 1964 Helsinki Declaration and its later amendments or comparable ethical standards. This study was approved by the Hospital Centre Sisters of Charity's Ethical Committee (251-29-11-20-01-5/ class 003-06/20-03/023).

Open Access This article is licensed under a Creative Commons Attribution 4.0 International License, which permits use, sharing, adaptation, distribution and reproduction in any medium or format, as long as you give appropriate credit to the original author(s) and the source, provide a link to the Creative Commons licence, and indicate if changes were made. The images or other third party material in this article are included in the article's Creative Commons licence, unless indicated otherwise in a credit line to the material. If material is not included in the article's Creative Commons licence and your intended use is not 
permitted by statutory regulation or exceeds the permitted use, you will need to obtain permission directly from the copyright holder. To view a copy of this licence, visit http://creativecommons.org/licenses/by/4.0/.

\section{References}

1. Kaplan FS, Pignolo RJ, Al Mukaddam MM, Shore EM (2017) Hard targets for a second skeleton: therapeutic horizons for fibrodysplasia ossificans progressiva (FOP). Expert Opin Orphan Drugs 5:291-294. https://doi.org/10.1080/21678707.2017.13042 11

2. Cappato S, Giacopelli F, Ravazzolo R, Bocciardi R (2018) The horizon of a therapy for rare genetic diseases: a "druggable" future for fibrodysplasia ossificans progressiva. Int J Mol Sci 19(4):989. https://doi.org/10.3390/ijms19040989

3. Kaplan FS, Pignolo RJ, Shore EM (2016) Granting immunity to FOP and catching heterotopic ossification in the act. Semin Cell Dev Biol 49:30-36. https://doi.org/10.1016/j.semcdb.2015.12.013

4. Matsuo K, Chavez RD, Barruet E, Hsiao EC (2019) Inflammation in fibrodysplasia ossificans progressiva and other forms of heterotopic ossification. Curr Osteoporos Rep 17:387-394. https://doi. org/10.1007/s11914-019-00541-x

5. Meyers C, Lisiecki J, Miller S, Levin A, Fayad L, Ding C, Sono T, McCarthy E, Levi B, James AW (2019) Heterotopic ossification: a comprehensive review. JBMR Plus 3:e10172. https://doi.org/10. 1002/jbm4.10172

6. Grgurević L, Novak R, Trkulja V, Hamzić LF, Hrkač S, Grazio S, Santini M (2019) Elevated plasma RANTES in fibrodysplasia ossificans progressiva - a novel therapeutic target? Med Hypotheses 131:109313. https://doi.org/10.1016/j.mehy.2019.109313

7. Rothan HA, Byrareddy SN (2020) The epidemiology and pathogenesis of coronavirus disease (COVID-19) outbreak. J Autoimmun 109:102433. https://doi.org/10.1016/j.jaut.2020.102433

8. Philips CA, Mohan N, Ahamed R, Kumbar S, Rajesh S, George T, Mohanan M, Augustine P (2020) One disease, many faces-typical and atypical presentations of SARS-CoV-2 infection-related COVID-19 disease. World J Clin Cases 8:3956-3970. https://doi. org/10.12998/wjcc.v8.i18.3956

9. Schneider CA, Rasband WS, Eliceiri KW (2012) NIH Image to ImageJ: 25 years of image analysis. Nat Methods 9:671-675. https://doi.org/10.1038/nmeth.2089

10. Gasparyan AY, Ayvazyan L, Blackmore H, Kitas GD (2011) Writing a narrative biomedical review: considerations for authors, peer reviewers, and editors. Rheumatol Int 31:1409-1417. https://doi. org/10.1007/s00296-011-1999-3

11. Scarlett RF, Rocke DM, Kantanie S, Patel JB, Shore EM, Kaplan FS (2004) Influenza-like viral illnesses and flare-ups of fibrodysplasia ossificans progressiva. Clin Orthop Relat Res 423:275-279. https://doi.org/10.1097/01.blo.0000129557.38803.26

12. Meyer C, Haustrate M-A, Nisolle JF, Deltombe T (2020) Heterotopic ossification in COVID-19: a series of 4 cases. Ann Phys Rehabil Med 63:565-567. https://doi.org/10.1016/j.rehab.2020. 09.010

13. Aziz A, Choudhari R, Alexander AJ, Allam E (2021) Heterotopic ossification post COVID-19: report of two cases. Radiol Case Rep 16:404-409. https://doi.org/10.1016/j.radcr.2020.12.002

14. Lees-Shepard JB, Yamamoto M, Biswas AA, Stoessel SJ, Nicholas SAE, Cogswell CA, Devarakonda PM, Schneider MJ Jr, Cummins SM, Legendre NP, Yamamoto S, Kaartinen V, Hunter JW, Goldhamer DJ (2018) Activin-dependent signaling in fibro/adipogenic progenitors causes fibrodysplasia ossificans progressiva. Nat Commun 9:471. https://doi.org/10.1038/s41467-018-02872-2

15. Mulholland BS, Forwood MR, Morrison NA (2019) Monocyte chemoattractant protein-1 (MCP-1/CCL2) drives activation of bone remodelling and skeletal metastasis. Curr Osteoporos Rep 17:538-547. https://doi.org/10.1007/s11914-019-00545-7

16. Wintges K, Beil FT, Albers J, Jeschke A, Schweizer M, Claass B, Tiegs G, Amling M, Schinke T (2013) Impaired bone formation and increased osteoclastogenesis in mice lacking chemokine (C-C motif) ligand 5 (Ccl5). J Bone Miner Res Off J Am Soc Bone Miner Res 28:2070-2080. https://doi.org/10.1002/jbmr.1937

17. Silfversward CJ (2018) Effects of IL-4 and IL-13 on bone. Dissertation, University of Uppsala

18. Macias MP, Fitzpatrick LA, Brenneise I, McGarry MP, Lee JJ, Lee NA (2001) Expression of IL-5 alters bone metabolism and induces ossification of the spleen in transgenic mice. J Clin Invest 107:949-959. https://doi.org/10.1172/jci11232

19. Fajgenbaum DC, June CH (2020) Cytokine storm. N Engl J Med 383:2255-2273. https://doi.org/10.1056/NEJMra2026131

20. Nalbandian A, Sehgal K, Gupta A, Madhavan MV et al (2021) Post-acute COVID-19 syndrome. Nat Med 27:601-615. https:// doi.org/10.1038/s41591-021-01283-Z

Publisher's Note Springer Nature remains neutral with regard to jurisdictional claims in published maps and institutional affiliations. 